\title{
Dependence of magnetic field just inside the magnetopause on subsolar standoff distance: Global MHD results
}

\author{
WANG Ming ${ }^{1,2}$, LU JianYong ${ }^{1,2 *}$, LIU ZiQian $^{3,4} \&$ PEI ShiXin ${ }^{1}$ \\ ${ }^{1}$ College of Math \& Physics, Nanjing University of Information Science \& Technology, Nanjing 210044, China; \\ ${ }^{2}$ National Center for Space Weather, China Meteorology Administration, Beijing 100081, China; \\ ${ }^{3}$ Chinese Academy of Meteorological Sciences, Beijing 100081, China; \\ ${ }^{4}$ Graduate University of Chinese Academy of Sciences, Beijing 100049, China
}

Received September 5, 2011; accepted November 24, 2011; published online February 26, 2012

\begin{abstract}
The subsolar magnetopause is the boundary between the solar wind and the Earth's magnetosphere, where reduced solar wind dynamic pressure is equal to the magnetic pressure of the Earth's outer magnetosphere. We use a global magnetohydrodynamic (MHD) model to estimate the ratio $f$ of the compressed magnetic field just inside the subsolar magnetopause to the purely dipolar magnetic field. We also compare our numerical results to a similar work by Shue, which used Time History of Events and Macroscale Interactions during Substorms (THEMIS) data. Our results show that the ratio $f$ is linearly proportional to the subsolar magnetopause standoff distance $\left(r_{0}\right)$ for both the northward and southward interplanetary magnetic field, properties consistent with Shue but with a smaller proportionality constant. However, previous theoretical studies show that $f$ is nearly independent of the subsolar standoff distance. The global model results also show that $f$ is smaller for the southward Interplanetary Magnetic Field (IMF) under the same $r_{0}$, and that the proportionality constant for the southward IMF is larger than that for the northward IMF. Both conclusions agree with statistical results from observations by Shue.
\end{abstract}

magnetopause, subsolar standoff distance, solar wind, magnetosphere

Citation: Wang M, Lu J Y, Liu Z Q, et al. Dependence of magnetic field just inside the magnetopause on subsolar standoff distance: Global MHD results. Chin Sci Bull, 2012, 57: 1438-1442, doi: 10.1007/s11434-011-4961-6

Previous theoretical studies without consideration of the impact of the solar wind take the internal magnetic field of the earth as a purely dipolar field. In the sun-earth line, knowing the distance from the earth, we can calculate the magnetic field at this position, as follows [1]:

$$
B_{d 0}=-M / r^{3} \text {, }
$$

where $B_{d 0}$ is the magnetic field for a purely dipolar field, $M=8.1 \times 10^{25} \mathrm{Gs} \mathrm{cm}^{3}$ is a constant, and $r$ is the distance from the earth.

When the solar wind flows toward the earth around the magnetosphere, the solar wind dynamic pressure $D_{p}$ is reduced. The reduced dynamic pressure is equal to the magnetic pressure of the earth's magnetosphere at the subsolar magnetopause [2]:

*Corresponding author (email: lujy@cma.gov.cn)

$$
k D_{p}=B_{g 0}^{2} / 2 \mu_{0}
$$

where $k$ is the reduction factor of the solar wind dynamic pressure, $B_{g 0}$ is the compressed magnetic field just inside the subsolar magnetopause, and $\mu_{0}$ is the permeability in free space.

Typically one can assume that $k$ is a constant $(k=0.881)$; then, $B_{g 0}$ becomes a variable about solar wind dynamic pressure $D_{p} . B_{d 0}$ is a variable about the distance from the earth on the sun-earth line for a purely dipolar field, so we take a ratio $f$ of the magnetic field just inside the subsolar magnetopause $\left(B_{g 0}\right)$ to the purely dipolar magnetic field $\left(B_{d 0}\right)$, for quantifying the compression level of the magnetic field. That is [2],

$$
f=B_{g 0} / B_{d 0} \text {. }
$$

Previous theoretical studies with simplified magnetosphere models assumed that $f$ was nearly independent of $r_{0}$ 
[3-6]. They did not consider the process of magnetic reconnection in their models. Aubry et al. [7] first reported that the magnetopause moves inward when magnetic reconnection occurs for the southward interplanetary magnetic field (IMF). Sibeck et al. [8] and Holzer and Slavin [9] determined $f^{2} / k$ from eq. (2) with given observed $D_{p}$ and $r_{0}$ $[10,11]$. The individual values of $f$ in the above works were not directly obtained from observation data. Recently, Shue [2] determined $f$ for both southward and northward IMF directly, from Time History of Events and their Macroscopic Interactions during Substorms (THEMIS) in situ data. On the other hand, numerical global magnetospheric models are becoming increasingly invaluable tools for detailed dynamic studies of solar-magnetosphere coupling. In this work, we examine the ratio $f$ from global MHD model calculations, and then compare with Shue's result. Compared to empirical works from observation data, observation results are limited by averaging effects, whereas the numerical model can give an exact response for certain solar wind and IMF conditions. Another aspect of this work can be considered to achieve further validation of global modeling with the observation models in solar wind-magnetosphere interaction.

\section{Data and model}

In this section, we introduce our global model to calculate the interaction of solar wind and magnetosphere under typical solar wind conditions, along with a method to determine the subsolar standoff distance from simulation data.

\subsection{Global MHD model of Space Weather Modeling Framework (SWMF)}

We use the Space Weather Modeling Framework (SWMF), developed by University of Michigan, to simulate the interaction between solar wind and magnetosphere [12]. The SWMF consists of several numerical modules, such as the ideal MHD solver (BATS-R-US) [13,14], Ionospheric Electrodynamics (IE) model [15], and Rice Convection Model (RCM) [16]. The SWMF family of models has been used extensively to study various solar wind influences on the magnetosphere; for example, convection under the northward IMF [17], IMF $B_{Y}$ [18], and Parker spiral [19] conditions and storm dynamics [20,21]. Welling and Ridley [22] discussed validation of the SWMF magnetic field and plasma using satellite measurements. Rae et al. [23] compared locations of observed and modeled open/closed field line boundaries, further demonstrating the effectiveness and reliability of SWMF. Lu et al. [24] constructed a three dimensional magnetopause shape model from global MHD results of SWMF [25,26].

In a typical simulation here, the computational domain is defined by $-70 R_{E} \leqslant X \leqslant 20 R_{E} ;-60 R_{E} \leqslant Y ; Z \leqslant 60 R_{E}$, with the grid size of $1.25 R_{E}$. Inside $-40 R_{E} \leqslant X \leqslant 20 R_{E} ;-45 R_{E} \leqslant Y$;
$Z \leqslant 45 R_{E}$ the grid size is $0.625 R_{E}$ and inside $-25 R_{E} \leqslant X \leqslant$ $12.5 R_{E} ;-30 R_{E} \leqslant Y ; Z \leqslant 30 R_{E}$, grid size is $0.3125 R_{E}$. The inner boundary is a sphere of $2.5 R_{E}$.

In our calculation, we consider the following combinations of IMF $B_{Z}$ and solar wind dynamic pressure (45 datasets): $B_{Z}=10,7.5,5,2.5,0,-2.5,-5,-7.5,-10 \mathrm{nT}$, and $D_{p}=1,2,3,4,5 \mathrm{nPa}, \mathrm{IMF} B_{X}=B_{Y}=0 \mathrm{nT}$ and $0^{\circ}$ dipole tilt angle. The magnetopause search is done on the $Y_{\mathrm{GSM}} Z_{\mathrm{GSM}}$ plane, until $X_{\mathrm{GSM}}$ reaches $-40 R_{E}$.

\subsection{Determining subsolar standoff distance}

The magnetopause is an important surface in space physics theory where the $y$ component of the current density $J_{y}$ has its largest absolute value; the change gradient of the plasma density $r$ is the largest; the $x$ component of solar wind velocity $u_{x}$ changes direction when solar wind flows around the magnetopause; the $z$ component of the interplanetary magnetic field $B_{z}$ changes direction when the interplanetary magnetic field is southward. The subsolar standoff distance is the distance between the earth and subsolar magnetopause.

Here, we search for the subsolar standoff position from the 45 datasets, according to the $u_{x}$ change of direction when the solar wind flows around the magnetopause, as shown in Figure 1.

This figure shows the relationship between the $x$ component of solar wind velocity $u_{x}$ and the distance from earth, on the $X$ axis. The corresponding solar wind condition in this calculation is $B_{z}=5 \mathrm{nT}$, and $D_{p}=1 \mathrm{nPa}$. In the figure, each small circle represents one data point. We first select $y=0$ and $z=0$, then we array the data from small to large, by $x$. Finally, new data is selected by $x$ and $u_{x}$, as shown in the figure. We also see that when $x$ is larger than $16 R_{E}, u_{x}$ is constant at $350 \mathrm{~km} / \mathrm{s}$ because they still reflect conditions within the solar wind, and we use constant solar wind

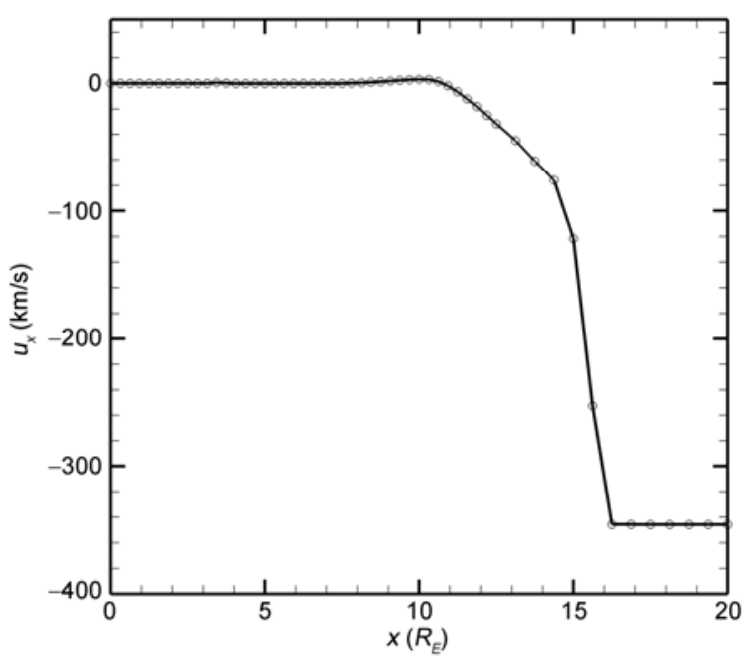

Figure 1 Relationship between $x$ component of solar wind velocity $u_{x}$ and distance from earth, on the $X$ axis. Corresponding solar wind condition is $B_{z}=5 \mathrm{nT}$, and $D_{p}=1 \mathrm{nPa}$. 
driving. At about $16 R_{E}, u_{x}$ suddenly drops, which indicates that the solar wind impacts the bow shock there. When $x$ is close to $10 R_{E}, u_{x}$ changes direction; this is the position of the subsolar magnetopause. In this example, the subsolar magnetopause surface is located at $x=10.625 R_{E}$. Considering the grid size, we approximate the closest grid point to the subsolar standoff magnetopause as the distance just inside the magnetopause. In this example, this point is $x=$ $10.3125 R_{E}$. Undoubtedly, this approximate but unavoidable selection of the distance just inside the subsolar magnetopause will produce an error and affect the qualitative result of $B_{g 0}, B_{d 0}$, and $f$.

Following the same procedure, we obtain all the positions just inside the magnetopause subsolar standoff distances in our global data sets from SWMF.

\section{Numerical results and discussion}

In this section, we present the calculation results of $B_{g 0}$ and $f$ for the southward and northward IMF in the data, and discuss their relation to the subsolar standoff distance.

\subsection{Relationship between $r_{0}$ and $B_{g 0}$}

Figure 2 shows the relationship between the compressed magnetic field just inside the subsolar magnetopause $B_{g 0}$ and the subsolar standoff distance $r_{0}$, for the northward and southward IMF. The dipolar magnetic field $B_{d 0}$ was calculated at the same position where $B_{g 0}$ was calculated from the data. The corresponding solar wind condition in this calculation is $B_{z}=5,10,-5,-10 \mathrm{nT}$, and $D_{p}=1,3,5 \mathrm{nPa}$.

The upward triangles represent $B_{g 0}$ in global MHD simulation data, and downward triangles are $B_{d 0}$ at the same position. The solid line is the result of fitting the relationship between $B_{g 0}$ and $r_{0}$. From Figure 2, we see that the compressed magnetic field just inside the subsolar magnetopause $B_{g 0}$ is linearly proportional to the subsolar standoff distance $r_{0}$, for the northward and southward IMF. $B_{g 0}$ is always larger than $B_{d 0}$ for the same $r_{0} . B_{g 0}$ for the northward IMF is larger than for the southward IMF, with the same $r_{0}$.

Figure 3 shows the relationship between the logarithm of the compressed magnetic field just inside the subsolar magnetopause $\ln B_{g 0}$ and the logarithm of the subsolar distance $\ln r_{0}$, for the northward and southward IMF. The corresponding solar wind condition in this calculation is $B_{z}=5$, $10,-5,-10 \mathrm{nT}$, and $D_{p}=1,3,5 \mathrm{nPa} . \ln r_{0}$ and $\ln B_{g 0}$ are calculated from the data. The solid line is the result of the fit. We see from the figure that the logarithm of the compressed magnetic field just inside the subsolar magnetopause $\ln B_{g 0}$ is strongly linearly proportional to the logarithm of the subsolar standoff distance $\ln r_{0}$, for the northward and southward IMF. $\ln B_{g 0}$ for the northward IMF is larger than for the southward IMF, with the same $\ln r_{0}$.

As in previous studies, we determine the compressed

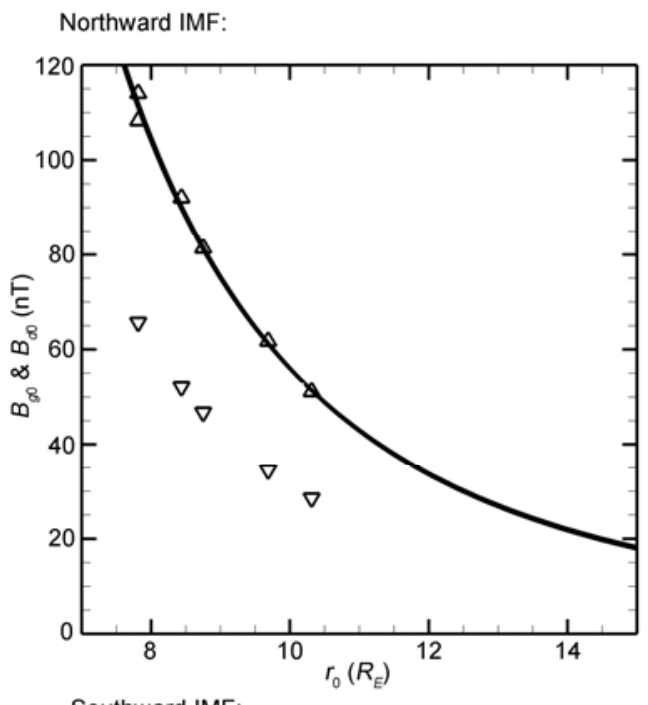

Southward IMF:

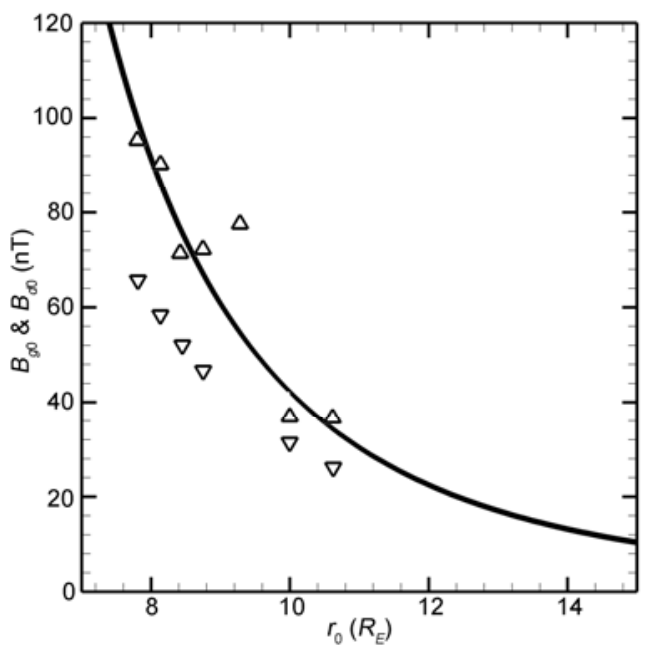

Figure 2 Relationship between compressed magnetic field just inside the subsolar magnetopause $B_{g 0}$ and the subsolar standoff distance $r_{0}$, for the northward and southward IMF. Dipolar magnetic field $B_{d 0}$ was calculated at the same position where $B_{g 0}$ was calculated from the data. Corresponding solar wind condition in this calculation is $B_{z}=5,10,-5,-10 \mathrm{nT}$, and $D_{p}=$ $1,3,5 \mathrm{nPa}$. Upward triangles represent $B_{g 0}$ in global simulation data, and downward triangles represent $B_{d 0}$. Solid line is the result of fitting the relationship between $B_{g 0}$ and $r_{0}$.

magnetic field just inside the subsolar magnetopause $B_{g 0}$ from $r_{0}$, using the following formula:

$$
B_{g 0}=A r_{0}{ }^{B} \text {, }
$$

where $A$ and $B$ are parameters of the function. By taking the logarithm of both sides, we rewrite eq. (4) in linear form:

$$
\ln B_{g 0}=\ln A+B \ln r_{0} .
$$

Then, we fit the terms $\ln B_{g 0}$ and $\ln r_{0}$. For the northward IMF, the result is

$$
\ln B_{g 0}=10.45-2.79 \ln r_{0} .
$$

Thus, we have $A=34544$ and $B=-2.79$. Eq. (4) can be rewritten as

$$
B_{g 0}=34544 r_{0}{ }^{-2.79} \text {. }
$$

Using the same method, we obtain the result for the 

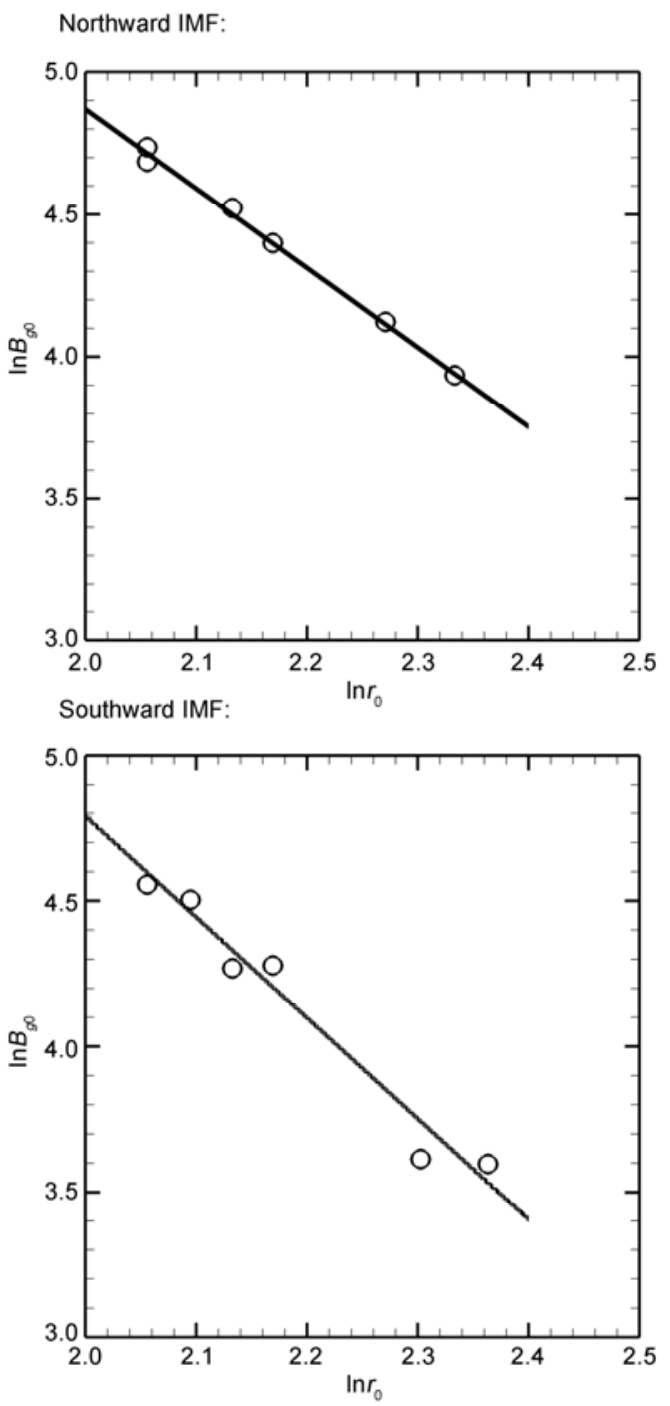

Figure 3 Relationship between logarithm of the compressed magnetic field just inside the subsolar magnetopause $\ln B_{g 0}$ and logarithm of the subsolar distance $\ln r_{0}$, for the northward and southward IMF. Corresponding solar wind condition in this calculation is $B_{z}=5,10,-5,-10 \mathrm{nT}$, and $D_{p}=1,3,5 \mathrm{nPa} . \ln r_{0}$ and $\ln B_{g 0}$ are calculated from the data. Solid line is result of fit.

southward IMF:

$$
\ln B_{g 0}=11.71-3.46 \ln r_{0} .
$$

That is, eq. (4) can be rewritten as

$$
B_{g 0}=121783 r_{0}^{-3.46} \text {. }
$$

\subsection{Relationship between $r_{0}$ and $f=B_{g 0} / B_{d 0}$}

Based on the above results, we further examine the relationship between $f=B_{g 0} / B_{d 0}$ and $r_{0}$.

Figure 4 shows $f$ as a function of subsolar standoff distance $r_{0}$. The corresponding solar wind condition in this calculation is $B_{z}=5,10,-5,-10 \mathrm{nT}$, and $D_{p}=1,3,5 \mathrm{nPa}$. The solid line is the result of the fit. From Figure 4, we see that $f$ of the compressed magnetic field just inside the subsolar magnetopause to the purely dipolar magnetic field is
Northward IMF:

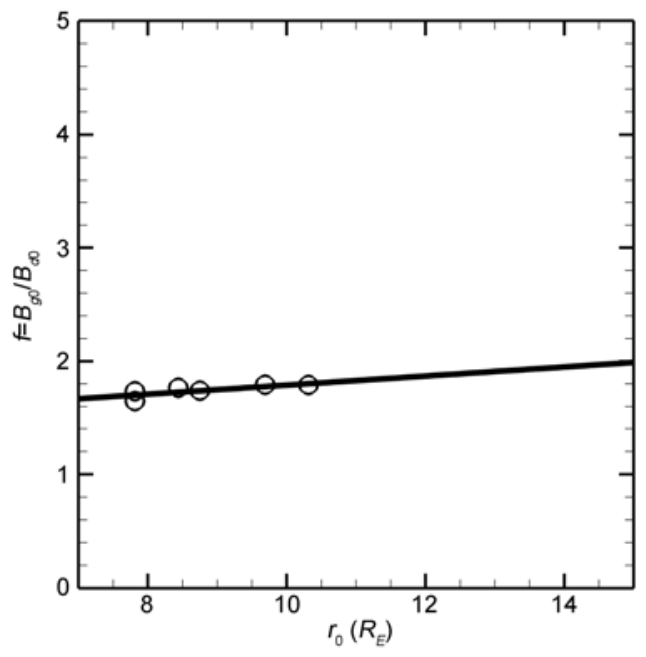

Southward IMF:

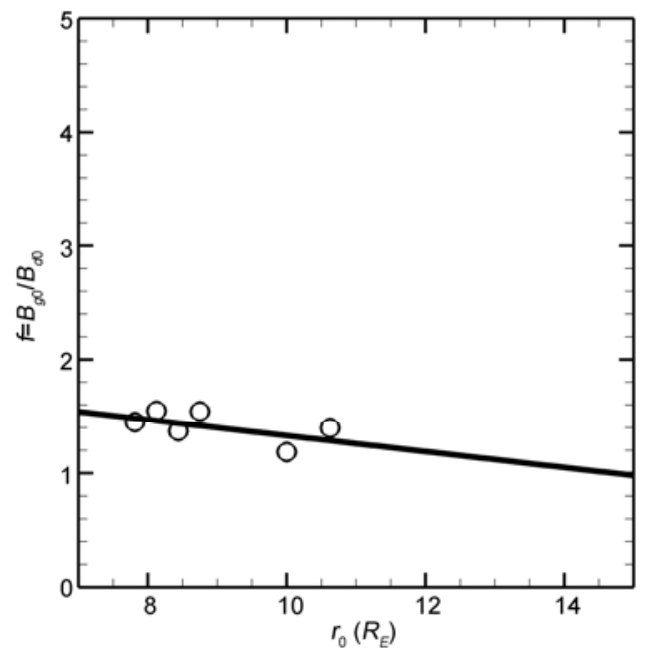

Figure 4 Ratio $(f)$ of the compressed magnetic field just inside the subsolar magnetopause $B_{g 0}$ to the dipolar magnetic field $B_{d 0}$, for the northward and southward IMF. Corresponding solar wind condition in this calculation is $B_{z}=5,10,-5,-10 \mathrm{nT}$, and $D_{p}=1,3,5 \mathrm{nPa}$. Solid line is result of fit.

linearly proportional to the subsolar standoff distance $r_{0}$, for both the northward and southward IMF. $f$ for the northward IMF is larger than for the southward IMF, with the same $r_{0}$.

For the northward IMF, the data fit gives

$$
f=0.04 r_{0}+1.39 \text {. }
$$

For the southward IMF, the data fit gives

$$
f=-0.07 r_{0}+2.03 \text {. }
$$

Compared with the results of Shue's, we find that (1) The finding that the ratio $f$ is linearly proportional to $r_{0}$ is consistent with Shue, but our $f$ values are small. This difference is caused by the selection of the point just inside the subsolar magnetopause standoff distance. We have no alternative to this, except to reduce the grid sizes in the global simulation of solar wind-magnetosphere coupling, which is not easily accomplished at present.

(2) From eqs. (10) and (11), we find that $f$ for the south- 
ward IMF is smaller than for the northward IMF, with the same $r_{0}$. This implies that the compression level of the magnetic field by the inward magnetopause for the southward IMF is smaller than for the northward IMF. This result agrees with Shue [2].

(3) The previous theoretical studies show that $f$ is nearly independent of the subsolar standoff distance. In our numerical results, the linear relationship between $f$ and the subsolar standoff distance $r_{0}$ exists, but its linear proportion is not strong. The proportional constants are small compared to those of Shue.

\section{Summary}

We use a global MHD model, the Space Weather Modeling Framework (SWMF), to estimate the ratio $f$ of the compressed magnetic field just inside the subsolar magnetopause $B_{g 0}$ to the purely dipolar magnetic field $B_{d 0}$. Model data are for various fixed solar wind conditions, and it helps capture the precise response of the magnetosphere to a specific solar wind condition, and distinguishes the effect of IMF direction.

We first determine subsolar standoff distance $r_{0}$, according to the $u_{x}$ change of direction when solar wind flows around the magnetopause. Then, using the model data, we calculate $B_{g 0}$ and $f$ for the northward and southward IMF. Then, we examine the relationship between $f$ and the subsolar standoff distance $r_{0}$. Finally, we compare our results with Shue. We find that: (1) The ratio of the magnetic field just inside the magnetopause to the purely dipolar magnetic field at that location is linearly proportional to the subsolar standoff distance; (2) The ratio $f$ for the southward IMF is smaller than for the northward IMF, and the compression level of the magnetic field by the inward magnetopause for the southward IMF is smaller than for the northward IMF.

In our next work, we will further investigate the relationship of $f$ and the subsolar standoff distance, including extreme solar wind conditions, such as those causing magnetic storms and substorms.

Simulation results were obtained using BATS-R-US, developed by the Center for Space Environment Modeling at the University of Michigan. This work was partially supported by the National Natural Science Foundation of China (40874087 and 41031063), the China Meteorology Administration (GYHY201106011) and the China Public Science and Technology Research Funds Projects of Ocean (201005017).

1 Barraclough D R, Mundt W, Barker F S, et al. International geomagnetic reference field revision 1985. Geophys J, 1986, 85: 217-220

2 Shue J H. Uneven compression levels of Earth's magnetic fields by shocked solar wind. J Geophys Res, 2010, 116: A02203

3 Choe J Y, Beard D B. The compressed geomagnetic field as a function of dipole tilt. Planet Space Sci, 1974, 22: 595-608
4 Ferraro V C A. An approximate method of estimating the size and shape of the stationary hollow carved out in a neutral ionized stream of corpuscles impinging on the geomagnetic field. J Geophys Res, 1960, 65: 3951-3953

5 Mead G D. Deformation of the geomagnetic field by the solar wind. J Geophys Res, 1964, 69: 1181-1195

6 Schield M A. Pressure balance between solar wind and magnetosphere. J Geophys Res, 1969, 74: 1275-1286

7 Aubry M P, Russell C T, Kivelson M G. Inward motion of the magnetopause before a substorm. J Geophys Res, 1970, 75: 7018-7031

8 Sibeck D G, Lopez R E, Roelof E C. Solar wind control of the magnetopause shape, location and motion. J Geophys Res, 1991, 96: 5489-5495

9 Holzer R E, Slavin J A. Magnetic flux transfer associated with expansions and contractions of the dayside magnetosphere. J Geophys Res, 1978, 83: 3831-3839

10 Zhao $\mathrm{H}$, Zong Q G, Wei Y, et al. Influence of solar wind dynamic pressure on geomagnetic Dst index during various magnetic storms. Sci China Tech Sci, 2011, 54: 1445-1454

11 Wei Y, Zong Q G, Pu Z Y, et al. Enhanced anti-sunward flow near local noon during a period of horizontal IMF and high solar wind velocity VY. Chin Sci Bull, 2011, 56: 1117-1122

12 Toth G, Sokolov I V, Gombosi T I, et al. Space Weather Modelling Framework: A new tool for the space science community. J Geophys Res, 2005, 110: A12226

13 De Zeeuw D L, Sazykin S, Wolf R A, et al. Coupling of a global MHD code and an inner magnetospheric model: Initial results. J Geophys Res, 2004, 109: A12219

14 Powell K G, Roe P L, Linde T J, et al. A solution-adaptive upwind scheme for ideal magnetohydrodynamics. J Comput Phys, 1999, 154: 284-309

15 Ridley A J, Hansen K C, Toth G, et al. University of Michigan MHD results of the Geospace Global Circulation Model metrics challenge. J Geophys Res, 2002, 107: A10,1290

16 Toffoletto F, Sazykin S, Spiro R, et al. Inner magnetospheric modeling with the Rice Convection Model. Space Sci Rev, 2003, 107: 175-196

17 Song P, DeZeeuw D L, Gombosi T I, et al. A numerical study of solar wind-magnetosphere interaction for northward interplanetary magnetic field. J Geophys Res, 1999, 104: 28361

18 Kabin K, Rankin R, Rostoker G, et al. Open-closed field line boundary position: A parametric study using an MHD model. J Geophys Res, 2004, 109: A05222

19 Gombosi T I, DeZeeuw D L, Groth C P T, et al. Magnetospheric configuration for Parker-spiral IMF conditions: Results of a 3D AMR MHD simulation. Adv Space Res, 2000, 26: 139-149

20 Toth G, De Zeeuw D L, Gombosi T I, et al. Sun-to-thermosphere simulation of the 28-30 October 2003 storm with the Space Weather Modelling Framework. Space Weather, 2007, 5: S06003

21 Zhang J, Liemohn M W, De Zeeuw D L, et al. Understanding storm-time ring current development through data-model comparisons of a moderate storm. J Geophys Res, 2007, 112: A04208

22 Welling D T, Ridley A J. Validation of SWMF magnetic field and plasma. Space Weather, 2010, 8: S03002

23 Rae I J, Kabin K, Lu J Y, et al. Comparison of the open-closed Separatrix in a global magnetospheric simulation with observations: The role of the ring current. J Geophys Res, 2010, 115: A08216

24 Lu J Y, Liu Z Q, Kabin K, et al. Three dimensional shape of the magnetopause: Global MHD results. J Geophys Res, 2011, 116: A09237

25 Lin R L, Zhang X X, Liu S Q, et al. Comparison of a new model with previous models for the low-latitude magnetopause size and shape. Chin Sci Bull, 2010, 55: 179-187

26 Li L, Feng Y Y. Energetic electron flux distribution model in the inner and middle magnetosphere. Sci China Tech Sci, 2011, 54: $441-446$

Open Access This article is distributed under the terms of the Creative Commons Attribution License which permits any use, distribution, and reproduction in any medium, provided the original author(s) and source are credited. 